Article

\title{
Immunopathogenesis of Emerging Candida auris and Candida haemulonii Strains
}

\author{
Sujiraphong Pharkjaksu ${ }^{1}$, Nawarat Boonmee ${ }^{2}$, Chalermchai Mitrpant ${ }^{2} \mathbb{D}$ and Popchai Ngamskulrungroj ${ }^{1, *} \mathbb{D}$ \\ 1 Department of Microbiology, Faculty of Medicine Siriraj Hospital, Mahidol University, \\ 10700 Bangkok, Thailand; sujiraphong.pha@mahidol.edu \\ 2 Department of Biochemistry, Faculty of Medicine Siriraj Hospital, Mahidol University, \\ 10700 Bangkok, Thailand; nawarat.boo@mahidol.ac.th (N.B.); chalermchai.mit@mahidol.edu (C.M.) \\ * Correspondence: popchai.nga@mahidol.ac.th; Tel.: +66-2-419-7069; Fax.: +66-2-411-3106
}

Citation: Pharkjaksu, S.; Boonmee, N.; Mitrpant, C.; Ngamskulrungroj, P. Immunopathogenesis of Emerging Candida auris and Candida haemulonii Strains. J. Fungi 2021, 7, 725. https://doi.org/10.3390/jof7090725

Academic Editor: Scott G. Filler

Received: 2 August 2021

Accepted: 1 September 2021

Published: 5 September 2021

Publisher's Note: MDPI stays neutral with regard to jurisdictional claims in published maps and institutional affiliations.

Copyright: (C) 2021 by the authors Licensee MDPI, Basel, Switzerland. This article is an open access article distributed under the terms and conditions of the Creative Commons Attribution (CC BY) license (https:// creativecommons.org/licenses/by/ $4.0 /)$.

\begin{abstract}
The emergence of a multidrug-resistant Candida species, C. auris and C. haemulonii, has been reported worldwide. In Thailand, information on them is limited. We collected clinical isolates from Thai patients with invasive candidiasis. Both species were compared with a laboratory C. albicans strain. In vitro antifungal susceptibility and thermotolerance, and pathogenesis in the zebrafish model of infection were investigated. Both species demonstrated high minimal inhibitory concentrations to fluconazole and amphotericin B. Only C. auris tolerated high temperatures, like C. albicans. In a zebrafish swim-bladder-inoculation model, the $C$. auris-infected group had the highest mortality rate and infectivity, suggesting the highest virulence. The case fatality rates of C. auris, C. haemulonii, and C. albicans were $100 \%, 83.33 \%$, and $51.52 \%$, respectively. Further immunological studies revealed that both emerging Candida species stimulated genes involved in the proinflammatory cytokine group. Interestingly, the genes relating to leukocyte recruitment were downregulated only for C. auris infections. Almost all immune response genes to $C$. auris had a peak response at an early infection time, which contrasted with $C$. haemulonii. In conclusion, both emerging species were virulent in a zebrafish model of infection and could activate the inflammatory pathway. This study serves as a stepping stone for further pathogenesis studies of these important emerging species.
\end{abstract}

Keywords: emerging Candida; innate immune response; multidrug resistance; zebrafish

\section{Introduction}

The Candida species are important causes of bloodstream infections in hospitalized patients. This is especially the case in intensive care units, where patients receive broadspectrum antimicrobial drugs, indwelling vascular catheters, parenteral nutrition, abdominal surgery, and immunosuppressive agents [1,2]. High mortality rates among patients have been associated with a delayed initiation of appropriate antifungal treatment [3,4]. This problem is compounded by the drug-resistant Candida, notably C. glabrata, found in many hospitals $[5,6]$.

An emerging multidrug-resistant Candida, C. auris, was first reported in 2009 as an isolate from the external ear at a hospital in Japan [7]. In 2011, it was found in a bloodstream infection in Korea [8]. During the last decade, approximately 39 countries in East Asia, the Middle East, Africa, North America, South America, and Europe reported cases of C. auris infection [9]. C. auris might be resistant to multiple classes of antifungal agents, such as echinocandins and azoles. Moreover, it has the potential for person-to-person transmission [10]. Conventional microbiological methods often misidentify C. auris as C. haemulonii, a phylogenetically related drug-resistant Candida species that is also being increasingly reported in hospitals worldwide [11]. The first clinical isolation of C. haemulonii was collected from the hemoculture of a patient with renal failure which was reported by Lavarde et al. in 1984 [12]. A few years ago, treatment failures for C. haemulonii infections 
were associated with unresponsiveness to amphotericin B; reduced susceptibility to azoles and echinocandins has also been reported [13-15].

Considering the importance of these emerging human pathogens, it is imperative to understand the host defense mechanisms. However, the mechanisms regarding C. auris and C. haemulonii responses after infection are largely unknown [16]. Host defense against Candida species relies on an interaction between the innate and adaptive immune responses. Firstly, there is a physical barrier, consisting of the skin and mucosa. The second barrier is recognition of the Candida species by innate immune cells, such as neutrophils, monocytes, and macrophages. The recognition is driven by fungal pathogen-associated molecular patterns, which are mainly associated with fungal cell walls such as $\beta$-glucan or phospholipomannan (PLM) [17,18]. The subsequent release of proinflammatory cytokines, combined with the antigen-presentation activity of myeloid cells, is crucial for shaping the adaptive immunity, which represents a long-term barrier against fungal infection [19]. Recent studies showed that $C$. auris infection led to reduced neutrophil activities and macrophage lysis capacity $[20,21]$. In comparison to Candida albicans, a stronger cytokine response was also observed in $C$. auris which mediated through the recognition of C-type lectin receptors. Collectively, C. auris was less virulent than C. albicans in in vivo experimental models of disseminated candidiasis [21]. However, studies of host immune response to C. haemulonii was still lacking.

Some studies on bacteria have reported finding an association between the antibiotic resistance and host pathogenesis [22,23]. In our study, we investigated the interaction between emerging Candida strains, C. auris and C. haemulonii, exhibiting high MICs to antifungal agents and host immune response by using zebrafish as a model for an immunopathogenesis study. Upon finishing this work, we found that C. auris and C. haemulonii were both highly virulent and several proinflammatory cytokine genes were involved in their pathogenesis. This highlights the advantage of using the zebrafish model to determine the virulence of Candida species, and potentially to investigate these emerging drug-resistant Candida species in the future.

\section{Materials and Methods}

\subsection{Clinical Isolates}

C. auris and C. haemulonii isolates were collected from the yeast culture collection held by the Mycology Unit, Department of Microbiology, Faculty of Medicine Siriraj Hospital, Mahidol University, Thailand. The isolates had been obtained from invasive candidiasis patients at the hospital, firstly in 2018 (from blood tested for the C. auris strain SI-18CAU-HEM) and later in 2021 (from pleural effusion tested for the $C$. haemulonii strain SI-21-CH-PLF).

Species identification was confirmed by ITS sequencing (ATCG Company Ltd., Thailand). The ITS sequences were compared to reference sequences deposited in the GenBank Databases (https:/ / blast.ncbi.nlm.nih.gov accessed on 28 May 2021). Accurate species identification targets included an $E$-value of $\leq 10^{5}$ and identity and coverage of $\geq 98 \%$ [24].

The nucleotide sequences of $C$. auris and $C$. haemulonii were assigned NCBI database accession numbers MZ312603 and MZ312604, respectively, and the ITS phylogenetic tree was represented in Figure S1. Before commencement of this research, its protocol was approved by the ethics committee of the Siriraj Institutional Review Board (Si. 091/2016; 9 February 2021).

\subsection{Thermotolerance and Antifungal Susceptibility Testing}

Assessment of thermotolerance was performed by spotting serial dilutions of C. auris and C. haemulonii, plus the control strain, C. albicans ATCC24433, on Sabouraud dextrose agar (SDA) plates and assessing growth after $48 \mathrm{~h}$ incubation at $30^{\circ} \mathrm{C}, 37^{\circ} \mathrm{C}$, and $42{ }^{\circ} \mathrm{C}$.

The susceptibility of the yeast strains to antifungal drugs was determined by using Sensititre YeastOne YO10 (SYO; Thermo Fisher Scientific, Waltham, MA, USA), a colorimetric microdilution method, as per the manufacturer's instructions. Nine drugs were 
used: fluconazole, voriconazole, itraconazole, posaconazole, 5-flucytosine, anidulafungin, micafungin, caspofungin, and amphotericin $\mathrm{B}$.

\subsection{Zebrafish Maintenance and Infection Experiment}

Adult wild-type zebrafish (Danio rerio, Tuebingen/AB strain) were kept in recirculated water aquarium under an alternated light/dark cycle of $14 \mathrm{~h}$ and $10 \mathrm{~h}$, respectively. Larval zebrafish were incubated at $28.5^{\circ} \mathrm{C}$ in $\mathrm{E} 3$ buffer $(60 \times$ stock solution: $34.8 \mathrm{~g} \mathrm{NaCl}, 1.6 \mathrm{~g} \mathrm{KCl}$, $5.8 \mathrm{~g} \mathrm{CaCl}_{2} \cdot 2 \mathrm{H}_{2} \mathrm{O}, 9.78 \mathrm{~g} \mathrm{MgCl}_{2} \cdot 6 \mathrm{H}_{2} \mathrm{O}$ with $100 \mu \mathrm{L}$ of $1 \%$ methylene blue $[\mathrm{MB}]$ in working solution). For the infection experiments, the larvae were manually dechorionated between 24- and 30-hour post-fertilization. Prior to microinjection, the larvae were anesthetized in E3-MB containing $0.2 \mathrm{mg} / \mathrm{mL}$ tricaine (ethyl-3-aminobenzoate; Sigma-Aldrich) [25].

The yeast strains were cultured in yeast-peptone-dextrose agar (YPD; Becton Dickinson, Franklin Lakes, NJ, USA). The plated cultures were inoculated to $5 \mathrm{~mL}$ liquid YPD and grown for $18 \mathrm{~h}$ at $30^{\circ} \mathrm{C}$ in a shaking incubator at $250 \mathrm{rpm}$. Yeast cells were centrifuged at $8000 \times g$, washed twice with phosphate buffer saline (PBS), and resuspended in $2 \mathrm{~mL}$ PBS. The concentration of the yeast suspension was adjusted by counting under a light microscope with a hemacytometer (INCYTO C-CHIP, Korea). This diluted suspension was pelleted at $8000 \times g$ for $15 \mathrm{~min}$ and resuspended in autoclaved $10 \%$ polyvinylpyrrolidone 40 (PVP40) with $0.5 \%$ phenol red in PBS [26].

The yeast cells were inoculated to zebrafish larvae 5 days post-fertilization (dpf) using a microinjector. Three nanoliters of the yeast suspension (adjusted to 150 and 250 yeast cells) were injected into the swim bladders of the zebrafish larvae [27]. Mock injected control and infected zebrafish larvae were kept in E3 buffer at $30^{\circ} \mathrm{C}$. This study was approved by the Siriraj Animal Care and Use Committee (SiACUC) (020/2562; 27 January 2021).

\subsection{Survival Rate}

The survival study was performed using 26 larvae per group. Survival of infected zebrafish larvae was recorded daily up to day nine.

\subsection{Infectivity Assessment}

Infectivity was determined by colony forming unit (CFU) quantification in biological triplications, based on minor adaptation of a previous study [28]. Five representative infected larvae were pooled and homogenized at $8 \mathrm{hpi}$ (hour-post-infection), $24 \mathrm{hpi}$, and 96 hpi in $50 \mu \mathrm{L}$ of $1 \mathrm{X}$ PBS. For plating, $20 \mu \mathrm{L}$ of homogenate from each group were plated on YPD agar supplemented with antibiotics (penicillin/streptomycin). To achieve a countable number of colonies, homogenate (undiluted sample), 1:10, 1:100, 1:1000, and 1:10000 dilutions were plated for sample at each time point. Plates were incubated overnight at 30 ${ }^{\circ} \mathrm{C}$, and colonies were counted the following day. Biological triplication was undertaken to ensure consistency of the experimental results.

\subsection{RNA Extraction and Expression Analysis}

RNA was isolated from 8 and 96 hpi larvae by using RNeasy Mini Kit (Qiagen, Hilden, Germany) following the manufacturer's instructions. To synthesize cDNA, total of $50 \mathrm{ng}$ of RNA were used in a reverse transcription reaction by using iScript Reverse Transcriptase (Bio-Rad, USA).

Each $20 \mu \mathrm{L}$ reaction mixture of real time PCR contained $5 \mu \mathrm{L}$ of $40 \mathrm{ng}$ cDNA, 50 $\mathrm{nM}$ concentrations of each gene-specific primer (Table S1), and $10 \mu \mathrm{L}$ of LightCycler 480 SYBR Green I Master Mix (Roche Life Science, Penzberg, Germany). Real-time PCR was performed on a Roche LightCycler 480 machine. cDNA quantitation was performed in triplicates, and reactions were normalized against the $\beta$-actin gene as an internal control [22]. cDNA was amplified with an initial denaturation at $95^{\circ} \mathrm{C}$ for $10 \mathrm{~min}$ before 40 cycles of denaturation $\left(95^{\circ} \mathrm{C}\right.$ for $\left.10 \mathrm{~s}\right)$, annealing $\left(52-58^{\circ} \mathrm{C}\right.$ for $\left.20 \mathrm{~s}\right)$, and extension $\left(72{ }^{\circ} \mathrm{C}\right.$ for $\left.20 \mathrm{~s}\right)$. This was then followed by a melting curve and cooling step. Determination of expression was calculated by normalized expression ratio $\left(2^{-\Delta \Delta C T}\right)$ compared with the $\beta$-actin gene. 


\subsection{Statistical Analysis}

Statistical analyses were performed using GraphPad Prism 8. In vivo in zebrafish data were assessed with the Mantel-Cox test to determine survival analysis. CFU quantifications and normalized gene expressions at different timepoints were compared by ordinary oneway ANOVA with Tukey's multiple comparison test. In all cases, $p<0.05$ was deemed significant.

\section{Results}

\subsection{Antifungal Susceptibility and Thermotolerant Testing}

The MICs of the C. auris and C. haemulonii strains investigated in this study are presented in Table 1. Compared with C. albicans ATCC24433, these emerging Candida strains showed high MICs for fluconazole: the MIC of $C$. auris was 512 times higher, while that of $C$. haemulonii was 64 times greater than the $C$. albicans strain. Moreover, the $C$. auris strain had high MICs for other azoles and amphotericin B.

Table 1. Antifungal susceptibility test results of the C. albicans (control strain), C. auris, and C. haemulonii strains.

\begin{tabular}{cccccccccccc}
\hline Strain & \multicolumn{7}{c}{ Minimum Inhibitory Concentration (MIC, ug/mL) } \\
\cline { 2 - 10 } & AND & MF & CAS & AB & 5FC & PZ & VOR & IZ & FZ \\
\hline $\begin{array}{c}\text { C. albicans } \\
\text { ATCC24433 } \\
\text { C. auris }\end{array}$ & $\leq 0.015$ & $\leq 0.008$ & 0.015 & 0.12 & $\leq 0.06$ & 0.03 & 0.015 & $\leq 0.015$ & 0.5 \\
$\begin{array}{c}\text { SI-18-CAU-HEM } \\
\text { C. haemulonii } \\
\text { SI-21-CH-PLF }\end{array}$ & 0.12 & 0.12 & 0.25 & 4 & 0.25 & $\geq 8$ & $\geq 8$ & $\geq 16$ & $\geq 256$ \\
\hline
\end{tabular}

Abbreviations: 5FC, 5-flucytosine; AB, amphotericin B; AND, anidulafungin; CAS, caspofungin; MF, micafungin; IZ, itraconazole; FZ, fluconazole; PZ, posaconazole; VOR, voriconazole.

Growth at a physiological temperature is a prerequisite for microbial invasion and pathogenicity. The $\mathrm{C}$. haemulonii isolates grew well at $30^{\circ} \mathrm{C}$, but their growth was poor or absent at $37^{\circ} \mathrm{C}$, and no growth occurred at $42^{\circ} \mathrm{C}$. In contrast, while the C. auris and C. albicans isolates were able to grow in temperatures ranging from $30^{\circ} \mathrm{C}$ to $42^{\circ} \mathrm{C}$, C. auris demonstrated better growth than C. albicans (Figure 1).

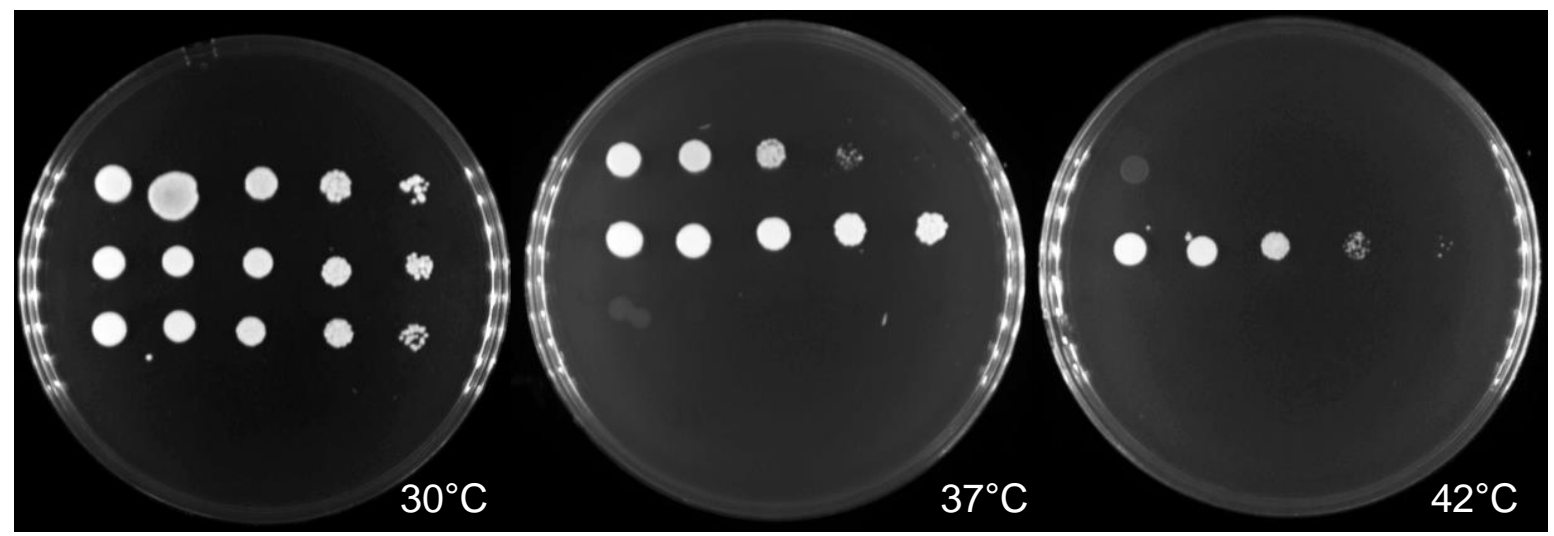

Figure 1. Differing thermotolerances of Candida albicans, C. auris, and C. haemulonii. Sabouraud dextrose agar plates showing growth of representative Candida strains after $48 \mathrm{~h}$ incubation at $30-42{ }^{\circ} \mathrm{C}$ with serial dilution spots. Top row: C. albicans ATCC24433 as the control strain; middle row: C. auris strain SI-18-CAU-HEM; and bottom row: C. haemulonii strain SI-21-CH-PLF. 


\subsection{Survival in a Zebrafish Model of a Mucosal Candida Infection at the Swim Bladder}

To evaluate the virulences of the C. albicans, C. auris, and C. haemulonii strains in a zebrafish model of a mucosal Candida infection at the swim bladder, their survival was monitored over the course of nine days after infection (Figure 2). Although inoculation with the C. albicans strain resulted in 50\% zebrafish death but the difference was not significant compared to the control group with $10 \%$ zebrafish death $(p=0.251)$. Interestingly, inoculation with $C$. auris produced $100 \%$ zebrafish death $(p<0.0001)$, whereas $C$. haemulonii produced $80 \%$ zebrafish death $(p=0.004)$ when comparted to the death in the control group. This result indicates that the $C$. auris and $C$. haemulonii strains had more virulence than the C. albicans strain.

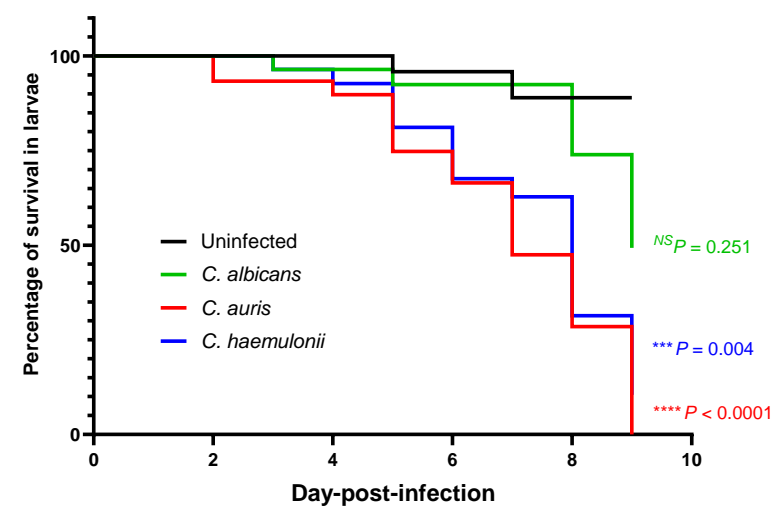

Figure 2. Emerging Candida virulence in a zebrafish model. Each experimental group of 26 zebrafish was injected with $10^{7} \mathrm{CFU}$ cells of one of the C. albicans ATCC24433 (control) strain, C. auris strain SI-18-CAU-HEM, or C. haemulonii strain SI-21-CH-PLF. Each experiment was performed in duplicate.

\subsection{Fungal Burden in Candidiasis Zebrafish Model}

The ability to colonize within a host is essential for Candida infections. For this reason, a CFU was used to examine Candida within the zebrafish. As illustrated in Figure 3, the fungal burden in all groups of fish was counted at different timepoints. The number of Candida cells significantly increased from 24 to 96 hpi in all groups $(p>0.0001)$, which indicated that there was cell proliferation. In particular, at $96 \mathrm{hpi}$, the number of cells in fish injected with $C$. auris was the highest, followed by $C$. haemulonii and C. albicans. This correlated with the survival analysis results.

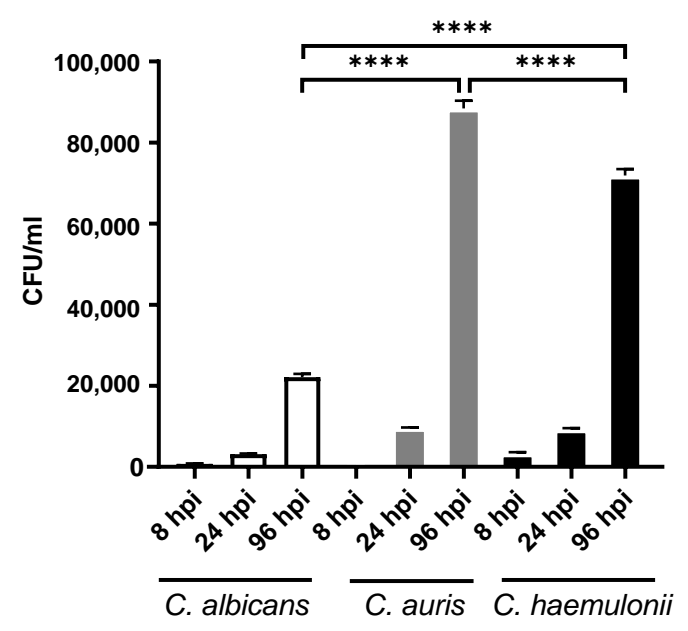

Figure 3. Fungal burden in zebrafish model. Each experimental group of five zebrafish was injected with $10^{7}$ cells of one of the C. albicans ATCC24433 (control) strain, C. auris strain SI-18-CAU-HEM, and C. haemulonii strain SI-21-CH-PLF. Each experiment was performed in triplicate. Abbreviations and symbols: CFU/mL, colony forming unit per milliliter; ${ }^{* * * *}, p<0.0001$. 


\subsection{Dynamics of Immune Response Genes to Emerging Candida in Zebrafish Model}

During an infection with Candida, the host immune system recognizes the pathogenassociated molecular patterns and induces the expression of cytokines [29]. To examine the pathogen-zebrafish interactions, the expression profiles of the host immune response genes that played an important role to activate immune cells and secrete cytokines or other components during Candida infection were monitored by real-time quantitative PCR (qPCR) at 8 hpi (as the early timepoint) and 96 hpi (as the late timepoint). The raw data of normalized gene expression was shown in Table S2 and Figure S2. The fold changes of the gene expression levels in zebrafish infected with $C$. auris and $C$. haemulonii were compared with the expression in zebrafish infected with the C. albicans control strain (Table 2).

Table 2. Fold changes of gene expression levels of zebrafish infected with C. auris and C. haemulonii at different timepoints, compared with C. albicans infection.

\begin{tabular}{|c|c|c|c|c|}
\hline \multirow{2}{*}{ Genes } & \multicolumn{2}{|c|}{ Early Timepoint (8 hpi) } & \multicolumn{2}{|c|}{ Late Timepoint (96 hpi) } \\
\hline & C. auris & C. haemulonii & C. auris & C. haemulonii \\
\hline \multicolumn{5}{|c|}{ Proinflammatory and Inflammatory Cytokines } \\
\hline $\operatorname{tnfa}$ & $3.74^{* * * *}$ & $2.60 *$ & $2.13^{*}$ & $2.06^{*}$ \\
\hline ifng & $1.22^{N S}$ & $1.27^{N S}$ & $0.92^{N S}$ & $1.76^{N S}$ \\
\hline$i l 1 b$ & $2.43^{* * *}$ & $1.22^{N S}$ & $1.12^{N S}$ & $1.96^{* *}$ \\
\hline$i l 6$ & $0.97^{N S}$ & $1.25^{N S}$ & $0.63^{* *}$ & $1.05^{N S}$ \\
\hline$i l 8$ & $4.72 * * * *$ & $2.37^{* * *}$ & $2.05^{N S}$ & $2.51 * *$ \\
\hline$i l 10$ & $2.47^{* *}$ & $1.35^{N S}$ & $1.33^{N S}$ & $2.00 * *$ \\
\hline$i l 17 a$ & $1.18^{N S}$ & $2.31^{* * *}$ & $1.25^{N S}$ & $0.78^{N S}$ \\
\hline \multicolumn{5}{|c|}{ Leukocyte Activities } \\
\hline inos & $1.07^{N S}$ & $0.93^{N S}$ & $0.96^{N S}$ & $0.85^{N S}$ \\
\hline$m p x$ & $0.98^{N S}$ & $0.83^{N S}$ & $0.42^{* * * *}$ & $1.04^{N S}$ \\
\hline \multicolumn{5}{|c|}{ Matrix Metalloproteinases } \\
\hline mmp9 & $0.76^{N S}$ & $3.82 * * * *$ & $2.09^{* * * *}$ & $2.41^{* * * *}$ \\
\hline mmp13 & $1.62 *$ & $1.72 *$ & $1.74 *$ & $1.88^{* *}$ \\
\hline \multicolumn{5}{|c|}{ Inflammatory Regulators } \\
\hline myd88 & $1.54^{N S}$ & $1.06^{N S}$ & $0.92^{N S}$ & $1.05^{N S}$ \\
\hline$n f k b$ & $1.02^{N S}$ & $1.56^{* *}$ & $1.17^{N S}$ & $2.11^{* * * *}$ \\
\hline$j a k 2$ & $3.04^{* * * *}$ & $0.46^{*}$ & $0.93^{N S}$ & $0.52^{N S}$ \\
\hline stat3 & $0.87^{N S}$ & $1.17^{N S}$ & $1.23^{N S}$ & $1.53 *$ \\
\hline \multicolumn{5}{|c|}{ Regulatory T-Cells } \\
\hline foxp3a & $3.16^{* * * *}$ & $1.12^{N S}$ & $0.33^{* * * *}$ & $0.92^{N S}$ \\
\hline foxp3b & $1.49 * *$ & $0.96^{N S}$ & $0.56^{* * * *}$ & $0.97^{N S}$ \\
\hline
\end{tabular}

Note: The expression folds were analyzed with the Livak method $\left(2^{-\Delta \Delta \mathrm{CT}}\right):<1$, downregulation; 1, basal; $>1$ upregulation. Abbreviations and symbols: hpi, hour post infection; ${ }^{N S}$, not significant; ${ }^{*}, p<0.5 ;{ }^{* *}, p<0.01$; $* * *, p<0.001 ;{ }^{* * * *}, p<0.0001$.

Most of the proinflammatory and inflammatory cytokine expression genes were upregulated in the $C$. auris and $C$. haemulonii infection. Compared with the C. albians group, the normalized ratios of expression were at significantly higher levels for both the $C$. auris and C. haemulonii groups (for example, $t n f a, i l 1 b$, ils, il10, and il17a). There was a high fold change of expression in C. auris group during the early timepoint, but the C. haemulonii group peaked at the late timepoint. Moreover, we found significant downregulation in il6 in the C. auris infection at the late phase (Table 2 and Table S3, Figure 4). The expressions of 
tnfa, il8, and il10 in the C. auris and C. haemulonii groups were at a higher level than in the C. albicans group at both the early and late timepoints (Figure S3).

il1b
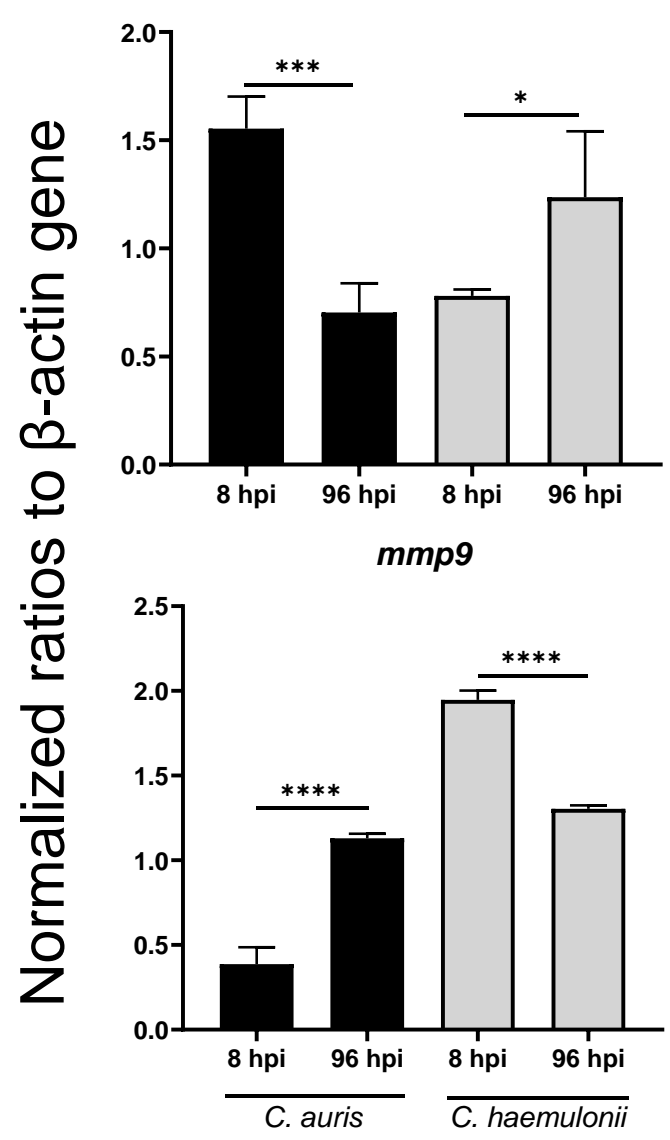

il10
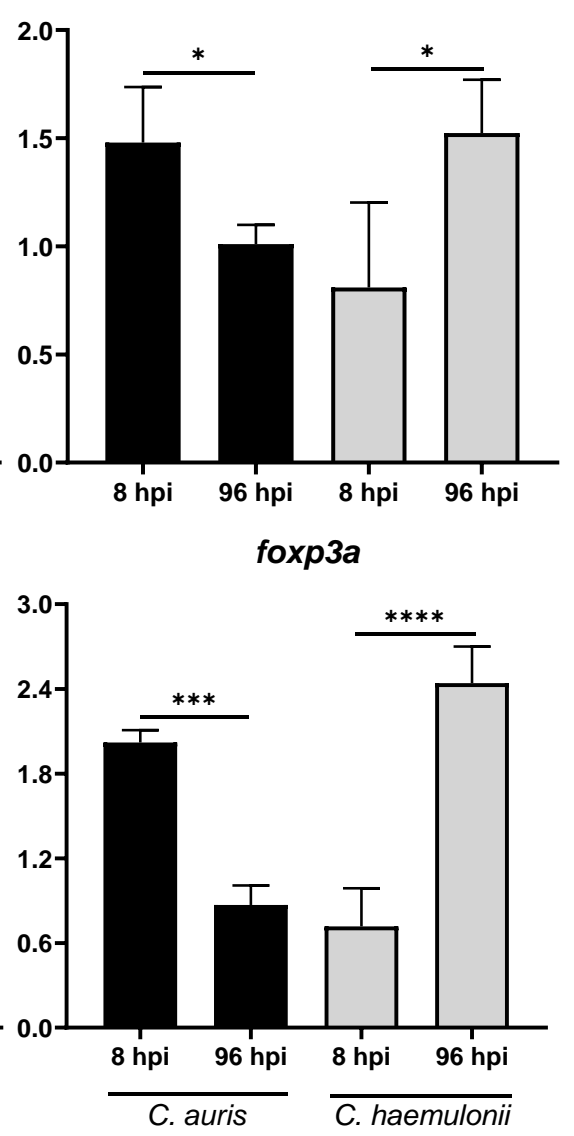

il17a
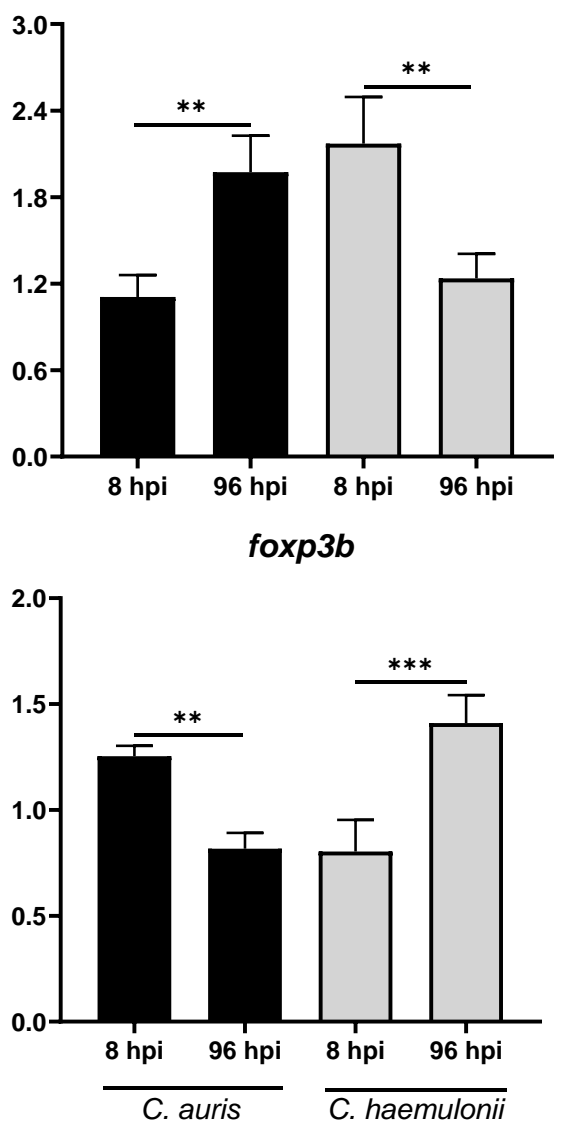

Figure 4. The expression levels of $i l 1 b$, il10, il17a, mmp9, foxp $3 a$, and foxp $3 b$ in zebrafish infected with emerging Candida strains, by timepoint. Each experimental group of 20 zebrafish was injected with $10^{7} \mathrm{CFU}$ cells of one of the $C$. auris strain SI-18-CAU-HEM or the C. haemulonii strain SI-21-CH-PLF. The normalized ratios of expression were calculated by comparison with the level of expression of the $\beta$-actin gene in each group at $8 \mathrm{hpi}$. Each experiment was performed in triplicate. Abbreviations and symbols: hpi, hour-post-infection; ${ }^{*}, p<0.5{ }^{* *}, p<0.01 ;{ }^{* * *}, p<0.001 ;{ }^{* * * *}, p<0.0001$.

To further understand the role of leukocytes and their activities to defend Candida, we determined the expression of inos (nitric oxide synthase in macrophage) and $m p x$ (myeloperoxidase in leukocytes). No significant differences in the expression levels of either inos and mpx were detected among the three groups at the early timepoint. Interestingly, the $m p x$ expression in the $C$. auris infection was significantly downregulated at the late timepoint. The results are illustrated in Table 2.

Next, the matrix metalloproteinase expressions as the leukocyte recruitment mediator [30] revealed that the matrix metalloproteinases genes were significantly upregulated in C. auris and C. haemulonii in both phases. Focusing on $m m p 9$, the C. auris infected group had a significantly high expression at the late phase, whereas the $C$. haemulonii group surged at the early phase (Figure 4). As to the inflammatory regulatory gene expression, most genes were activated at a similar level to that of the C. albicans infection, with the jak2 expression at a significantly increased level in C. auris at the early timepoint (Table 2). Interestingly, $n f k b$ demonstrated higher fold change expressions after the C. auris and C. haemulonii infections than the C. albicans group (Figure S3).

Moreover, we detected the gene expressions of foxp $3 a$ and foxp $3 b$, given their importance in the development and function of regulatory T-cells [31]. The results revealed that the C. haemulonii infection had expressions that were at a similar level to those of the C. albi- 
cans infection, while the $C$. auris infection showed a significantly high expression for both genes (Table 2). Additionally, we found a different timepoint of gene expression among the strains. The $C$. auris infection was significantly upregulated at the early timepoint, but the C. haemulonii infection increased at the late timepoint (Figure 4).

\section{Discussion}

The global emergence and spread of $C$. auris as a causative agent of invasive nosocomial infection has arisen from resistance to multiple antifungal drugs and possibly to all major classes of systemic antifungal drugs [5,32]. In addition, there has been horizontal transmission among hospitalized patients [32,33]. C. auris and C. haemulonii are phylogenetically related species in the Metschnikowiaceae family, and they have multidrug-resistance properties [34]. We studied the characteristics of the first-isolated C. auris and C. haemulonii at our hospital, and we highlighted experimental evidence to identify differences in their drug susceptibility patterns, pathogenicity, and host responses in zebrafish. C. albicans was used as the reference strain.

In our study, C. auris and C. haemulonii demonstrated high MICs for fluconazole and amphotericin B, while echinocandin MICs were within the susceptible range, according to the tentative MIC breakpoint value that had been previously established [13,35]. This result was in concordance with the findings of other studies, namely, that high MICs for triazoles and amphotericin B among emerging $C$. auris and $C$. haemulonii strains were increasingly apparent in clinical settings [11,35-40]. Thermotolerant testing revealed that $C$. albicans and $C$. auris - but not $C$. haemulonii-could grow at $30-42{ }^{\circ} \mathrm{C}$, and that $C$. auris had the highest thermotolerance. This corresponds with the findings of recent publications [41,42]. Our survival analysis showed that $C$. auris exhibited the highest mortality rate of infection, followed by $C$. haemulonii and C. albicans. As to the fungal burden in the experimental model of zebrafish, the $C$. auris with the highest thermotolerance had the highest infectivity.

Based on our findings, C. auris was more virulent and caused earlier mortality of infected larvae. This is consistent with other work, which reported that this species demonstrated more severity than C. haemulonii in animal studies $[43,44]$. One United Kingdom study reported that $C$. auris did not form cellular aggregates, thereby causing a significant virulence in terms of the mortality rate [45]. Additionally, the genomes of $C$. auris and C. haemulonii, which are closely related species, contain C. albicans gene orthologs, such as proteinases and mannosyl transferases, which might play roles in pathogenesis. However, the genes of $C$. albicans have not been characterized [46]. However, previous studues reported that $C$. auris and $C$. haemulonii were less virulent than other Candida species (such as C. albicans and C. tropicalis) $[41,47,48]$. This might be explained by the fact that $C$. auris and $C$. haemulonii lacked the ability to produce hyphae, which is an important virulence factor for disseminated infections. Therefore, a comparison between swim bladder, performed in this study, and intravenous route of infections would be required and warranted in the next study. An interesting factor in our study was the high MICs for antifungals of the emerging Candida isolates. This factor might cause increasing severity or virulence from pathogens, as presented by other studies on Staphylococcus aureus [49], Escherichai coli, Pseudomonas aeruginosa [23], and Vibrio alginolyticus [50].

To understand the pathogenesis of emerging Candida strains, we performed gene expression analysis to observe the host immune response to pathogens in systemic infections. Firstly, proinflammatory cytokines play a role in stimulating immune cells (especially macrophages) to destroy pathogens [51]. The expression of most proinflammatory cytokine genes was upregulated at the early phase with $C$. auris. Although a similar pattern was observed with C. haemulonii, some genes (such as il1b and il10) peaked at the late phase. On the other hand, Il10, an inflammatory cytokine, was induced in an early phase of $C$. auris infection. This inflammatory cytokine could potentially be released by Toll like receptor 2 (TLR2) dependent pathway in macrophage and hampered proinflammatory cytokines that is critical for neutrophil recruitment [52,53]. Interestingly, il17 $\alpha$ activation as a key to the cytokine gene that links to neutrophil recruitment [54] showed a slow response after C. auris 
infection and il8 and $m p x$, surrogate markers for neutrophil function, were significantly reduced in later phase of $C$. auris infection. This finding contrasted with $C$. albicans and C. haemulonii infection in that their responses occurred at the early timepoint. This result suggests that the neutrophil function in a host infected with $C$. auris is less capable than that of neutrophils in larvae infected with $C$. haemulonii and C. albicans and may partly be an explanation for virulence of $C$. auris.

Matrix metalloproteinase (MMPs) are members of the proteolytic enzyme family and play multiple roles in the normal immune response to infection, including leucocyte recruitment, cytokine, and chemokine processing, and defensin activation [55]. We found that the expression of $m m p 9$ had an opposing pattern to proinflammatory cytokine genes such as tnfa and il1b, which supports the role of MMPs in controlling the production of proinflammatory cytokines [56]. The key to proinflammatory cytokine production is the activation of the transcription factor, NFkB, after the toll-like receptors (TLRs)-pathogen interaction in innate immunity. Almost all TLRs signal via MyD88 as an adaptor protein for NFkB activation, with subsequent inflammatory cytokine production and control of adaptative immunity $[57,58]$. The data correlated to the previous description as the myd88/nfkb gene expressions were stimulated by pathogen. The levels of expression fluctuated due to different modes of activation, demonstrating that NF-kB activation is an important requirement for the expression of many Candida-regulated genes [59]. As to other transductors, JAK/STAT is the signal transduction pathway of many essential cytokines involved in sepsis [60].

Lastly, regulatory $\mathrm{T}\left(\mathrm{T}_{\text {reg }}\right)$ cells play a major role in the suppression of excessive immune responses. The functions of these cells were controlled by the expression of regulatory gene encoding the forkhead box P3 (FOXP3) protein $[61,62]$. Our results showed that Candida infection (other than C. auris) activated foxp3a and foxp $3 b$ at the late timepoint suggesting that $C$. auris strain might reduce the number of regulatory $T$ cells at the late time point and allow to increase yeast population compared to other Candida species. This data agreed with a previous study [63] that demonstrated the roles of foxp $3 a$ and foxp $3 b$ in suppressing inflammatory cytokine secretion and $\mathrm{T}$ cell maintenance in zebrafish. Moreover, that earlier research identified that foxp $3 a$ and foxp $3 b$ could stimulate the IL17-secreted cell response to Candida infection; this corresponded with the findings of the current study [64].

As mentioned earlier, the effects of drug resistant isolate might be influenced by the host immune response. Jiang JH et al. [49] recorded that the daptomycin-resistant Staphylococcus aureus strain had impaired neutrophil recruitment in vivo and promoted bacterial survival. Moreover, gene expression of proinflammatory cytokines and molecules of innate immunity (such as lysozyme and C3b in zebrafish larvae infected with ceftazidimeresistant [50] and levofloxacin-resistant [65] Vibrio alginolyticus strains) showed higher upregulation than susceptible strains.

In summary, the strains of emerging Candida species, C. auris, and C. haemulonii, with high MICs for antifungal agents, showed significantly higher virulences than the $C$. albicans control strain used in the zebrafish model. In terms of the immune response, differences in the patterns of gene expression were noted, especially for $C$. auris. The benefits of using a zebrafish model to study the pathogenesis of fungal infections were considered. Our results highlight the potential of using zebrafish as an effective model for the investigation of the mechanisms controlling infections as well as for therapeutic efficiency studies. As the number of strains in this study was limited due to very few cases at our hospital, we need to collect more strains for future research.

Supplementary Materials: The following are available online at https: / www.mdpi.com/article / 10.3390/jof7090725/s1. Table S1: Primers used for qPCR experiments in this study [22,28,63,66-69]; Table S2: Average of gene expression levels of zebrafish infected with $C$. auris and $C$. haemulonii at different timepoints, compared with the expression level of $\beta$-actin in each group; Figure S1: Phylogenic tree using ITS sequences of emerging strains in this study [70]; Figure S2: qPCR analysis of the gene expression levels in zebrafish infected with Candida albicans ATCC24433 as a control strain 
in this study; Figure S3: qPCR analysis indicating the high alterations of the expression levels of $\operatorname{tnfa}$, $i l 8, i l 10$, and $n f k b$ in zebrafish infected with emerging Candida strains.

Author Contributions: Conceptualization, C.M. and P.N.; methodology, S.P., C.M., and P.N.; software, S.P.; validation, S.P., C.M. and P.N.; formal analysis, S.P.; investigation, S.P. and N.B.; resources, S.P. and N.B.; data curation, S.P.; writing of original draft, S.P., C.M., and P.N.; review and editing of manuscript, S.P., C.M., and P.N.; visualization, S.P.; supervision, C.M. and P.N.; project administration, C.M. and P.N.; funding acquisition, P.N. All authors have read and agreed to the published version of the manuscript.

Funding: This research was funded by the Siriraj Research Fund, Faculty of Medicine Siriraj Hospital, Mahidol University (R016333017).

Institutional Review Board Statement: The study was conducted according to the guidelines of the Declaration of Helsinki and approved by the Institutional Review Board of Faculty of Medicine Siriraj Hospital, Mahidol University (Si. 091/2016; 9 February 2021). The animal ethics was approved by the Siriraj Animal Care and Use Committee (SiACUC) of the Faculty of Medicine Siriraj Hospital, Mahidol University (020/2562; 27 January 2021).

Informed Consent Statement: Not applicable.

Data Availability Statement: Not applicable.

Acknowledgments: We are very grateful to Nerisa Thornsri, Clinical Epidemiology Unit, Office for Research and Development, Faculty of Medicine Siriraj Hospital, Mahidol University for helping with the statistical analyses, as well as the staff of the Mycology Laboratory, Department of Microbiology, Faculty of Medicine Siriraj Hospital, Mahidol University for the reference and clinical strains used in our study. Moreover, Narisa Poonsub, Sukanya Ruchadaariyachat, and Wipada Phromkhuntod provided zebrafish husbandry and technical support.

Conflicts of Interest: All authors declare that there are no conflicts of interest related to this study. The funders had no role in the design of the study; in the collection, analyses, or interpretation of data; in the writing of the manuscript; or in the decision to publish the results.

\section{References}

1. Magill, S.S.; Edwards, J.R.; Bamberg, W.; Beldavs, Z.G.; Dumyati, G.; Kainer, M.A.; Lynfield, R.; Maloney, M.; McAllister-Hollod, L.; Nadle, J.; et al. Multistate point-prevalence survey of health care-associated infections. N. Engl. J. Med. 2014, 370, 1198-1208. [CrossRef]

2. Pappas, P.G.; Lionakis, M.S.; Arendrup, M.C.; Ostrosky-Zeichner, L.; Kullberg, B.J. Invasive candidiasis. Nat. Rev. Dis. Prim. 2018, 4, 18026. [CrossRef]

3. Garey, K.W.; Rege, M.; Pai, M.P.; Mingo, D.E.; Suda, K.J.; Turpin, R.S.; Bearden, D.T. Time to initiation of fluconazole therapy impacts mortality in patients with candidemia: A multi-institutional study. Clin. Infect. Dis. 2006, 43, 25-31. [CrossRef] [PubMed]

4. Andes, D.R.; Safdar, N.; Baddley, J.W.; Playford, G.; Reboli, A.C.; Rex, J.H.; Sobel, J.D.; Pappas, P.G.; Kullberg, B.J. Impact of treatment strategy on outcomes in patients with candidemia and other forms of invasive candidiasis: A patient-level quantitative review of randomized trials. Clin. Infect. Dis. 2012, 54, 1110-1122. [CrossRef]

5. Castanheira, M.; Messer, S.A.; Rhomberg, P.R.; Pfaller, M.A. Antifungal susceptibility patterns of a global collection of fungal isolates: Results of the SENTRY Antifungal Surveillance Program (2013). Diagn. Microbiol. Infect. Dis. 2016, 85, 200-204. [CrossRef]

6. Pfaller, M.A.; Moet, G.J.; Messer, S.A.; Jones, R.N.; Castanheira, M. Geographic variations in species distribution and echinocandin and azole antifungal resistance rates among Candida bloodstream infection isolates: Report from the SENTRY Antimicrobial Surveillance Program (2008 to 2009). J. Clin. Microbiol. 2011, 49, 396-399. [CrossRef] [PubMed]

7. Satoh, K.; Makimura, K.; Hasumi, Y.; Nishiyama, Y.; Uchida, K.; Yamaguchi, H. Candida auris sp. nov., a novel ascomycetous yeast isolated from the external ear canal of an inpatient in a Japanese hospital. Microbiol. Immunol. 2009, 53, 41-44. [CrossRef]

8. Lee, W.G.; Shin, J.H.; Uh, Y.; Kang, M.G.; Kim, S.H.; Park, K.H.; Jang, H.C. First three reported cases of nosocomial fungemia caused by Candida auris. J. Clin. Microbiol. 2011, 49, 3139-3142. [CrossRef] [PubMed]

9. Kean, R.; Brown, J.; Gulmez, D.; Ware, A.; Ramage, G. Candida auris: A Decade of Understanding of an Enigmatic Pathogenic Yeast. J. Fungi 2020, 6, 30. [CrossRef] [PubMed]

10. Ahmad, S.; Alfouzan, W. Candida auris: Epidemiology, Diagnosis, Pathogenesis, Antifungal Susceptibility, and Infection Control Measures to Combat the Spread of Infections in Healthcare Facilities. Microorganisms 2021, 9, 807. [CrossRef]

11. Lima, S.L.; Francisco, E.C.; de Almeida Júnior, J.N.; Santos, D.; Carlesse, F.; Queiroz-Telles, F.; Melo, A.S.A.; Colombo, A.L. Increasing Prevalence of Multidrug-Resistant Candida haemulonii Species Complex among All Yeast Cultures Collected by a Reference Laboratory over the Past 11 Years. J. Fungi 2020, 6, 110. [CrossRef] 
12. Lavarde, V. Peritonite mycosique a Torulopsis haemulonii. Bull. Soc. Fr. Mycol. Med. 1984, 13, $173-176$.

13. Cendejas-Bueno, E.; Kolecka, A.; Alastruey-Izquierdo, A.; Theelen, B.; Groenewald, M.; Kostrzewa, M.; Cuenca-Estrella, M.; Gómez-López, A.; Boekhout, T. Reclassification of the Candida haemulonii complex as Candida haemulonii (C. haemulonii group I), C. duobushaemulonii sp. nov. (C. haemulonii group II), and C. haemulonii var. vulnera var. nov.: Three multiresistant human pathogenic yeasts. J. Clin. Microbiol. 2012, 50, 3641-3651. [CrossRef] [PubMed]

14. Ruan, S.-Y.; Kuo, Y.-W.; Huang, C.-T.; Hsiue, H.-C.; Hsueh, P.-R. Infections due to Candida haemulonii: Species identification, antifungal susceptibility and outcomes. Int. J. Antimicrob. Agents 2010, 35, 85-88. [CrossRef]

15. Ramos, L.S.; Figueiredo-Carvalho, M.H.; Barbedo, L.S.; Ziccardi, M.; Chaves, A.L.; Zancopé-Oliveira, R.M.; Pinto, M.R.; Sgarbi, D.B.; Dornelas-Ribeiro, M.; Branquinha, M.H.; et al. Candida haemulonii complex: Species identification and antifungal susceptibility profiles of clinical isolates from Brazil. J. Antimicrob. Chemother. 2015, 70, 111-115. [CrossRef]

16. Lockhart, S.R.; Etienne, K.A.; Vallabhaneni, S.; Farooqi, J.; Chowdhary, A.; Govender, N.P.; Colombo, A.L.; Calvo, B.; Cuomo, C.A.; Desjardins, C.A.; et al. Simultaneous Emergence of Multidrug-ResistantCandida aurison 3 Continents Confirmed by Whole-Genome Sequencing and Epidemiological Analyses. Clin. Infect. Dis. 2017, 64, 134-140. [CrossRef]

17. Huffnagle, G.B.; Deepe, G.S. Innate and adaptive determinants of host susceptibility to medically important fungi. Curr. Opin. Microbiol. 2003, 6, 344-350. [CrossRef]

18. Romani, L. Immunity to fungal infections. Nat. Rev. Immunol. 2004, 4, 11-24. [CrossRef] [PubMed]

19. Richardson, J.P.; Moyes, D.L. Adaptive immune responses to Candida albicans infection. Virulence 2015, 6, 327-337. [CrossRef]

20. Johnson, C.J.; Davis, J.M.; Huttenlocher, A.; Kernien, J.F.; Nett, J.E. Emerging Fungal Pathogen Candida auris Evades Neutrophil Attack. mBio 2018, 9, e01403-18. [CrossRef]

21. Bruno, M.; Kersten, S.; Bain, J.M.; Jaeger, M.; Rosati, D.; Kruppa, M.D.; Lowman, D.W.; Rice, P.J.; Graves, B.; Ma, Z.; et al. Transcriptional and functional insights into the host immune response against the emerging fungal pathogen Candida auris. Nat. Microbiol. 2020, 5, 1516-1531. [CrossRef]

22. Bhuiyan, M.S.; Ellett, F.; Murray, G.L.; Kostoulias, X.; Cerqueira, G.M.; Schulze, K.E.; Mahamad Maifiah, M.H.; Li, J.; Creek, D.J.; Lieschke, G.J.; et al. Acinetobacter baumannii phenylacetic acid metabolism influences infection outcome through a direct effect on neutrophil chemotaxis. Proc. Natl. Acad. Sci. USA 2016, 113, 9599-9604. [CrossRef] [PubMed]

23. Cepas, V.; Soto, S.M. Relationship between Virulence and Resistance among Gram-Negative Bacteria. Antibiotics 2020, 9 , 719. [CrossRef]

24. Merseguel, K.B.; Nishikaku, A.S.; Rodrigues, A.M.; Padovan, A.C.; e Ferreira, R.C.; de Azevedo Melo, A.S.; Briones, M.R.; Colombo, A.L. Genetic diversity of medically important and emerging Candida species causing invasive infection. BMC Infect. Dis. 2015, 15, 57. [CrossRef]

25. Davis, J.M.; Huang, M.; Botts, M.R.; Hull, C.M.; Huttenlocher, A. A Zebrafish Model of Cryptococcal Infection Reveals Roles for Macrophages, Endothelial Cells, and Neutrophils in the Establishment and Control of Sustained Fungemia. Infect. Immun. 2016, 84, 3047-3062. [CrossRef]

26. Spaink, H.P.; Cui, C.; Wiweger, M.I.; Jansen, H.J.; Veneman, W.J.; Marin-Juez, R.; de Sonneville, J.; Ordas, A.; Torraca, V.; van der Ent, W.; et al. Robotic injection of zebrafish embryos for high-throughput screening in disease models. Methods 2013, 62, 246-254. [CrossRef] [PubMed]

27. Hachicho, N.; Reithel, S.; Miltner, A.; Heipieper, H.J.; Küster, E.; Luckenbach, T. Body Mass Parameters, Lipid Profiles and Protein Contents of Zebrafish Embryos and Effects of 2,4-Dinitrophenol Exposure. PLoS ONE 2015, 10, e0134755. [CrossRef]

28. Bergeron, A.C.; Seman, B.G.; Hammond, J.H.; Archambault, L.S.; Hogan, D.A.; Wheeler, R.T. Candida and Pseudomonas interact to enhance virulence of mucosal infection in transparent zebrafish. Infect. Immun. 2017. [CrossRef] [PubMed]

29. Netea, M.G.; Brown, G.D.; Kullberg, B.J.; Gow, N.A. An integrated model of the recognition of Candida albicans by the innate immune system. Nat. Rev. Microbiol. 2008, 6, 67-78. [CrossRef]

30. Xu, S.; Webb, S.E.; Lau, T.C.K.; Cheng, S.H. Matrix metalloproteinases (MMPs) mediate leukocyte recruitment during the inflammatory phase of zebrafish heart regeneration. Sci. Rep. 2018, 8, 7199. [CrossRef] [PubMed]

31. Fontenot, J.D.; Gavin, M.A.; Rudensky, A.Y. Foxp3 programs the development and function of CD4+CD25+ regulatory T cells. Nat. Immunol. 2003, 4, 330-336. [CrossRef]

32. Chowdhary, A.; Anil Kumar, V.; Sharma, C.; Prakash, A.; Agarwal, K.; Babu, R.; Dinesh, K.R.; Karim, S.; Singh, S.K.; Hagen, F.; et al. Multidrug-resistant endemic clonal strain of Candida auris in India. Eur. J. Clin. Microbiol. Infect. Dis. 2014, 33, 919-926. [CrossRef] [PubMed]

33. Chowdhary, A.; Sharma, C.; Duggal, S.; Agarwal, K.; Prakash, A.; Singh, P.K.; Jain, S.; Kathuria, S.; Randhawa, H.S.; Hagen, F.; et al. New clonal strain of Candida auris, Delhi, India. Emerg. Infect. Dis. 2013, 19, 1670-1673. [CrossRef] [PubMed]

34. Muñoz, J.F.; Gade, L.; Chow, N.A.; Loparev, V.N.; Juieng, P.; Berkow, E.L.; Farrer, R.A.; Litvintseva, A.P.; Cuomo, C.A. Genomic insights into multidrug-resistance, mating and virulence in Candida auris and related emerging species. Nat. Commun. 2018, 9 , 5346. [CrossRef]

35. Arendrup, M.C.; Prakash, A.; Meletiadis, J.; Sharma, C.; Chowdhary, A. Comparison of EUCAST and CLSI Reference Microdilution MICs of Eight Antifungal Compounds for Candida auris and Associated Tentative Epidemiological Cutoff Values. Antimicrob. Agents Chemother. 2017, 61, e00485-17. [CrossRef] 
36. Kathuria, S.; Singh, P.K.; Sharma, C.; Prakash, A.; Masih, A.; Kumar, A.; Meis, J.F.; Chowdhary, A. Multidrug-Resistant Candida auris Misidentified as Candida haemulonii: Characterization by Matrix-Assisted Laser Desorption Ionization-Time of Flight Mass Spectrometry and DNA Sequencing and Its Antifungal Susceptibility Profile Variability by Vitek 2, CLSI Broth Microdilution, and Etest Method. J. Clin. Microbiol. 2015, 53, 1823-1830. [CrossRef]

37. Chowdhary, A.; Prakash, A.; Sharma, C.; Kordalewska, M.; Kumar, A.; Sarma, S.; Tarai, B.; Singh, A.; Upadhyaya, G.; Upadhyay, S.; et al. A multicentre study of antifungal susceptibility patterns among 350 Candida auris isolates (2009-17) in India: Role of the ERG11 and FKS1 genes in azole and echinocandin resistance. J. Antimicrob. Chemother. 2018, 73, 891-899. [CrossRef] [PubMed]

38. Khan, Z.U.; Al-Sweih, N.A.; Ahmad, S.; Al-Kazemi, N.; Khan, S.; Joseph, L.; Chandy, R. Outbreak of fungemia among neonates caused by Candida haemulonii resistant to amphotericin B, itraconazole, and fluconazole. J. Clin. Microbiol. 2007, 45, $2025-2027$. [CrossRef] [PubMed]

39. Rodero, L.; Cuenca-Estrella, M.; Córdoba, S.; Cahn, P.; Davel, G.; Kaufman, S.; Guelfand, L.; Rodríguez-Tudela, J.L. Transient fungemia caused by an amphotericin B-resistant isolate of Candida haemulonii. J. Clin. Microbiol. 2002, 40, 2266-2269. [CrossRef] [PubMed]

40. Kim, S.; Ko, K.S.; Moon, S.Y.; Lee, M.S.; Lee, M.Y.; Son, J.S. Catheter-related candidemia caused by Candida haemulonii in a patient in long-term hospital care. J. Korean Med. Sci. 2011, 26, 297-300. [CrossRef] [PubMed]

41. Ben-Ami, R.; Berman, J.; Novikov, A.; Bash, E.; Shachor-Meyouhas, Y.; Zakin, S.; Maor, Y.; Tarabia, J.; Schechner, V.; Adler, A.; et al. Multidrug-Resistant Candida haemulonii and C. auris, Tel Aviv, Israel. Emerg. Infect. Dis. 2017, 23, 195-203. [CrossRef] [PubMed]

42. Reséndiz-Sánchez, J.; Ortiz-Álvarez, J.; Casimiro-Ramos, A.; Hernández-Rodríguez, C.; Villa-Tanaca, L. First report of a catheterrelated bloodstream infection by Candida haemulonii in a children's hospital in Mexico City. Int. J. Infect. Dis. 2020, 92, 123-126. [CrossRef] [PubMed]

43. Gandra, R.M.; McCarron, P.; Viganor, L.; Fernandes, M.F.; Kavanagh, K.; McCann, M.; Branquinha, M.H.; Santos, A.L.S.; Howe, O.; Devereux, M. In vivo Activity of Copper(II), Manganese(II), and Silver(I) 1,10-Phenanthroline Chelates Against Candida haemulonii Using the Galleria mellonella Model. Front. Microbiol. 2020, 11, 470. [CrossRef]

44. Muñoz, J.E.; Ramirez, L.M.; Dias, L.D.S.; Rivas, L.A.; Ramos, L.S.; Santos, A.L.S.; Taborda, C.P.; Parra-Giraldo, C.M. Pathogenicity Levels of Colombian Strains of Candida auris and Brazilian Strains of Candida haemulonii Species Complex in Both Murine and Galleria mellonella Experimental Models. J. Fungi 2020, 6, 104. [CrossRef]

45. Borman, A.M.; Szekely, A.; Johnson, E.M. Comparative Pathogenicity of United Kingdom Isolates of the Emerging Pathogen Candida auris and Other Key Pathogenic Candida Species. mSphere 2016, 1, e00189-16. [CrossRef] [PubMed]

46. Chatterjee, S.; Alampalli, S.V.; Nageshan, R.K.; Chettiar, S.T.; Joshi, S.; Tatu, U.S. Draft genome of a commonly misdiagnosed multidrug resistant pathogen Candida auris. BMC Genom. 2015, 16, 686. [CrossRef]

47. Fakhim, H.; Vaezi, A.; Dannaoui, E.; Chowdhary, A.; Nasiry, D.; Faeli, L.; Meis, J.F.; Badali, H. Comparative virulence of Candida auris with Candida haemulonii, Candida glabrata and Candida albicans in a murine model. Mycoses 2018, 61, 377-382. [CrossRef]

48. Sherry, L.; Ramage, G.; Kean, R.; Borman, A.; Johnson, E.M.; Richardson, M.D.; Rautemaa-Richardson, R. Biofilm-Forming Capability of Highly Virulent, Multidrug-Resistant Candida auris. Emerg. Infect. Dis. 2017, 23, 328-331. [CrossRef] [PubMed]

49. Jiang, J.H.; Bhuiyan, M.S.; Shen, H.H.; Cameron, D.R.; Rupasinghe, T.W.T.; Wu, C.M.; Le Brun, A.P.; Kostoulias, X.; Domene, C.; Fulcher, A.J.; et al. Antibiotic resistance and host immune evasion in Staphylococcus aureus mediated by a metabolic adaptation. Proc. Natl. Acad. Sci. USA 2019, 116, 3722-3727. [CrossRef]

50. Jiang, M.; Gong, Q.Y.; Lai, S.S.; Cheng, Z.X.; Chen, Z.G.; Zheng, J.; Peng, B. Phenylalanine enhances innate immune response to clear ceftazidime-resistant Vibrio alginolyticus in Danio rerio. Fish Shellfish. Immunol. 2019, 84, 912-919. [CrossRef]

51. Chin, V.K.; Foong, K.J.; Maha, A.; Rusliza, B.; Norhafizah, M.; Chong, P.P. Early expression of local cytokines during systemic Candida albicans infection in a murine intravenous challenge model. Biomed. Rep. 2014, 2, 869-874. [CrossRef]

52. Wagener, J.; Malireddi, R.K.; Lenardon, M.D.; Köberle, M.; Vautier, S.; MacCallum, D.M.; Biedermann, T.; Schaller, M.; Netea, M.G.; Kanneganti, T.D.; et al. Fungal chitin dampens inflammation through IL-10 induction mediated by NOD2 and TLR9 activation. PLoS Pathog. 2014, 10, e1004050. [CrossRef] [PubMed]

53. Netea, M.G.; Sutmuller, R.; Hermann, C.; Van der Graaf, C.A.; Van der Meer, J.W.; van Krieken, J.H.; Hartung, T.; Adema, G.; Kullberg, B.J. Toll-like receptor 2 suppresses immunity against Candida albicans through induction of IL-10 and regulatory T cells. J. Immunol. 2004, 172, 3712-3718. [CrossRef]

54. Zenobia, C.; Hajishengallis, G. Basic biology and role of interleukin-17 in immunity and inflammation. Periodontol. 2000 2015, 69, 142-159. [CrossRef] [PubMed]

55. Elkington, P.T.; O'Kane, C.M.; Friedland, J.S. The paradox of matrix metalloproteinases in infectious disease. Clin. Exp. Immunol. 2005, 142, 12-20. [CrossRef]

56. McQuibban, G.A.; Gong, J.H.; Wong, J.P.; Wallace, J.L.; Clark-Lewis, I.; Overall, C.M. Matrix metalloproteinase processing of monocyte chemoattractant proteins generates CC chemokine receptor antagonists with anti-inflammatory properties in vivo. Blood 2002, 100, 1160-1167. [CrossRef] [PubMed]

57. Kawai, T.; Akira, S. The role of pattern-recognition receptors in innate immunity: Update on Toll-like receptors. Nat. Immunol. 2010, 11, 373-384. [CrossRef] [PubMed]

58. Mogensen, T.H. Pathogen recognition and inflammatory signaling in innate immune defenses. Clin. Microbiol. Rev. 2009, 22, 240-273, Table of Contents. [CrossRef] [PubMed] 
59. Müller, V.; Viemann, D.; Schmidt, M.; Endres, N.; Ludwig, S.; Leverkus, M.; Roth, J.; Goebeler, M. Candida albicans triggers activation of distinct signaling pathways to establish a proinflammatory gene expression program in primary human endothelial cells. J. Immunol. 2007, 179, 8435-8445. [CrossRef]

60. Cai, B.; Cai, J.P.; Luo, Y.L.; Chen, C.; Zhang, S. The Specific Roles of JAK/STAT Signaling Pathway in Sepsis. Inflammation 2015, 38, 1599-1608. [CrossRef]

61. Hori, S.; Nomura, T.; Sakaguchi, S. Control of regulatory T cell development by the transcription factor Foxp3. Science 2003, 299, 1057-1061. [CrossRef]

62. Josefowicz, S.Z.; Lu, L.F.; Rudensky, A.Y. Regulatory T cells: Mechanisms of differentiation and function. Annu. Rev. Immunol. 2012, 30, 531-564. [CrossRef] [PubMed]

63. Sugimoto, K.; Hui, S.P.; Sheng, D.Z.; Nakayama, M.; Kikuchi, K. Zebrafish FOXP3 is required for the maintenance of immune tolerance. Dev. Comp. Immunol. 2017, 73, 156-162. [CrossRef] [PubMed]

64. Whibley, N.; Maccallum, D.M.; Vickers, M.A.; Zafreen, S.; Waldmann, H.; Hori, S.; Gaffen, S.L.; Gow, N.A.; Barker, R.N.; Hall, A.M. Expansion of Foxp3(+) T-cell populations by Candida albicans enhances both Th17-cell responses and fungal dissemination after intravenous challenge. Eur. J. Immunol. 2014, 44, 1069-1083. [CrossRef]

65. Jiang, M.; Yang, L.; Chen, Z.G.; Lai, S.S.; Zheng, J.; Peng, B. Exogenous maltose enhances Zebrafish immunity to levofloxacinresistant Vibrio alginolyticus. Microb. Biotechnol. 2020, 13, 1213-1227. [CrossRef] [PubMed]

66. Chao, C.C.; Hsu, P.C.; Jen, C.F.; Chen, I.H.; Wang, C.H.; Chan, H.C.; Tsai, P.W.; Tung, K.C.; Wang, C.H.; Lan, C.Y.; et al. Zebrafish as a model host for Candida albicans infection. Infect Immun 2010, 78, 2512-2521. [CrossRef]

67. Philip, A.M.; Wang, Y.; Mauro, A.; El-Rass, S.; Marshall, J.C.; Lee, W.L.; Slutsky, A.S.; dosSantos, C.C.; Wen, X.Y. Development of a zebrafish sepsis model for high-throughput drug discovery. Mol. Med. 2017, 23, 134-148. [CrossRef] [PubMed]

68. Zhang, C.N.; Zhang, J.L.; Huang, Y.; Ren, H.T.; Guan, S.H.; Zeng, Q.H. Dibutyltin depressed immune functions via NF-kB, and JAK/STAT signaling pathways in zebrafish (Danio rerio). Environ. Toxicol. 2018, 33, 104-111. [CrossRef] [PubMed]

69. Duan, J.; Ba, Q.; Wang, Z.; Hao, M.; Li, X.; Hu, P.; Zhang, D.; Zhang, R.; Wang, H. Knockdown of ribosomal protein S7 causes developmental abnormalities via p53 dependent and independent pathways in zebrafish. Int. J. Biochem. Cell Biol. 2011, 43, 1218-1227. [CrossRef]

70. Chybowska, A.D.; Childers, D.S.; Farrer, R.A. Nine Things Genomics Can Tell Us About Candida auris. Front. Genet. $2020,11,351$. [CrossRef] 